IMPLANT THERAPY OUTCOMES, SURGICAL ASPECTS

\section{Early loading of implants with acid-etched surface. A long-term clinical study}

Moreno J, Nuñez E, Ortiz I, España A, Jiménez A, Velasco E.

Master in Implant Dentistry. Faculty of Dentistry. University of Seville.

\section{Abstract}

Introduction and objectives. Implant dentistry is currently a dental procedure in patients with full and partial tooth loss. The aim of this study is to show the evaluation of patients with early loading of
implants with acid-etched surface. Methodology. 35 implants with acid-etched surface. Methodology. 35 patients with partial and total tooth loss were
treated with acid-etched surface Defcon TSA $\circledast$ implants. All implants were inserted in 1 surgery.
Implants were loaded after a healing period of 6 weeks in the jaw and 8 weeks in the upper jaw. Clinical findings (implants and prosthodontics) were
followed for 16 years. Results. 100 implants were followed for 16 years. Results. 100 implants were
inserted into both jaws (47 in the maxilla and 53 in inserted into both jaws (47 in the maxilla and 53 in
the mandible) for prosthodontic rehabilitation. 54 implants were restored with single crowns, 24 with fixed bridges and 22 implants with overdentures. The results indicate a survival and success rate of
implants of $95 \%$, after 16 years of clinical follow-up. During the healing period of functional loading, 3 implants were lost because of mobility. In 5 implants, it was observed peri-implantitis, two implant were lost. Conclusions. The clinical findings of this study indicate that prosthodontic rehabilitation with implants with acid-etched surface and early loaded, represents a successful dental treatment.

\section{Background and Aim}

The aim of this study is to show the evaluation of patients treated by early loading of implants with acid etched surface.
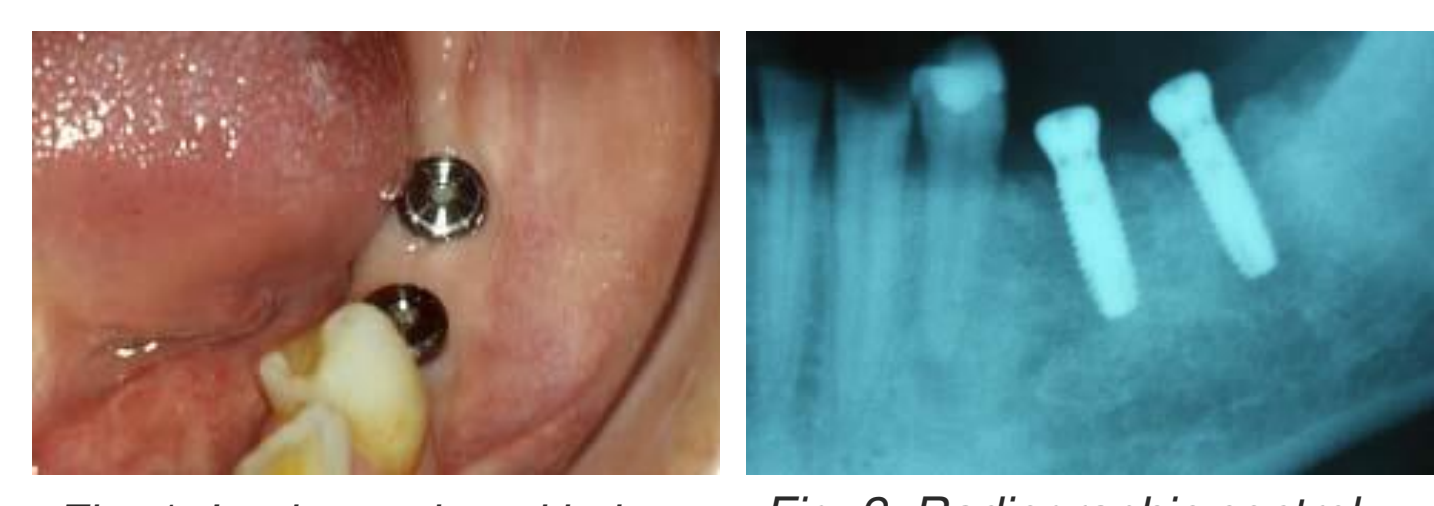

Fig. 1. Implants placed in jaw

Fig. 2. Radiographic control

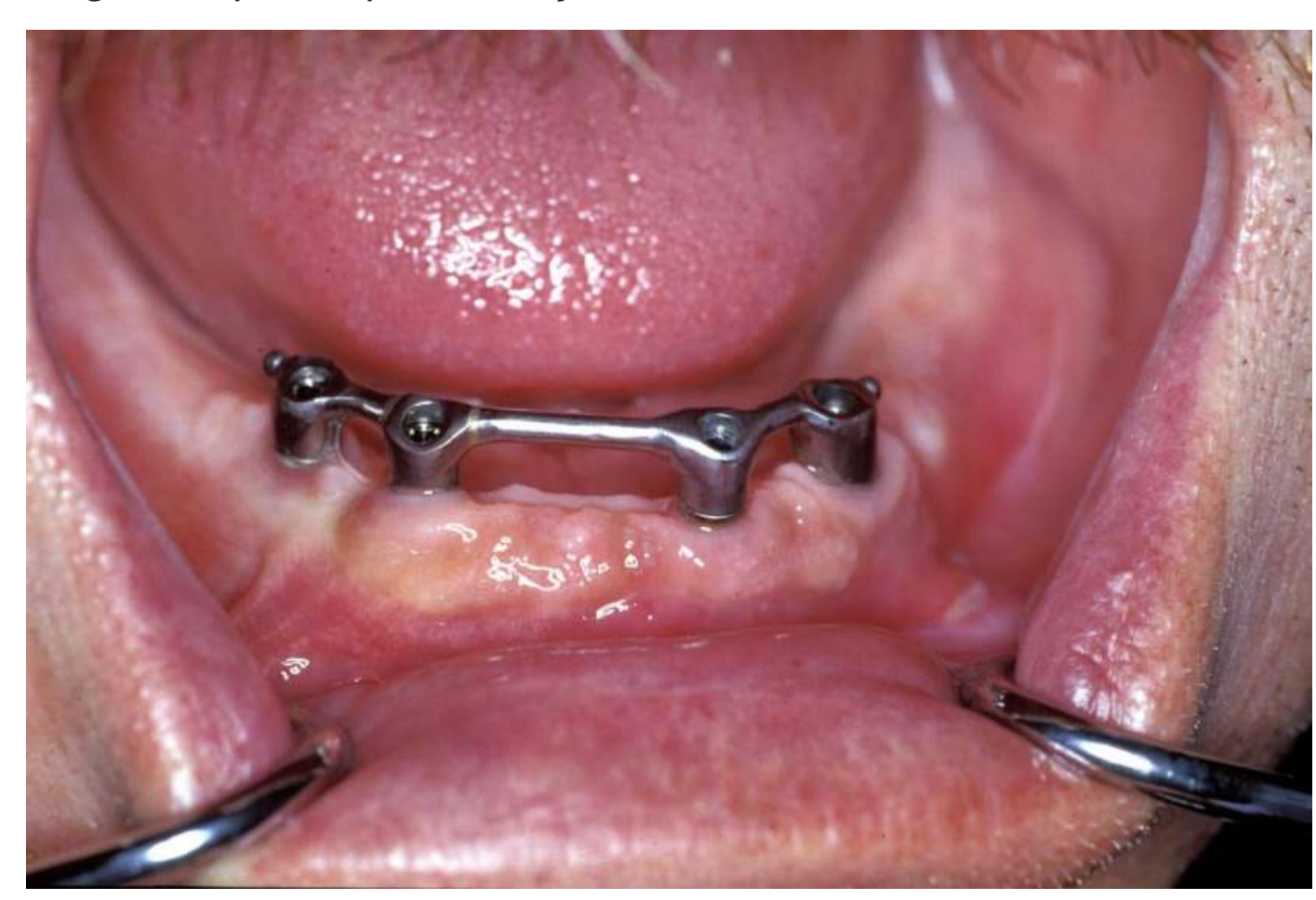

Fig. 3. Bar for overdenture

\section{Methods and Materials}

This study was conducted by professors from the Faculty of Dentistry at the University of Seville. Prior to the study, patients suffering from serious systemic disorders that could compromise osseointegration were excluded from the study. The selected patients were adults of both sexes. With regard to oral conditions, patients had no periodontal disease, no history of having suffered in the past. A very important requirement was the availability of a sufficient amount of alveolar bone in the jaws or edentulous sections. This study only included the realization of basic surgical procedures and more complex techniques (i.e. guided tissue regeneration, bone grafts, growth factors) were excluded.

All patients enrolled in the study were informed of the treatment protocol with implants, surgical and prosthetic aspects, timing of treatment and monitoring, as well as the possibility of the incidence of complications and loss of implants. Patients implant treatment authorized by a consent form. Before treatment, all patients were evaluated radiologically, with a panoramic radiograph, also when it was necessary periapical radiographs and CT scans were performed. The criteria for success and survival of the implants were recommended by van Steenberghe et al ${ }^{1}$. In fact, survival was defined as the proportion of permanent implants in their original location even without clinical value or cause adverse effects.
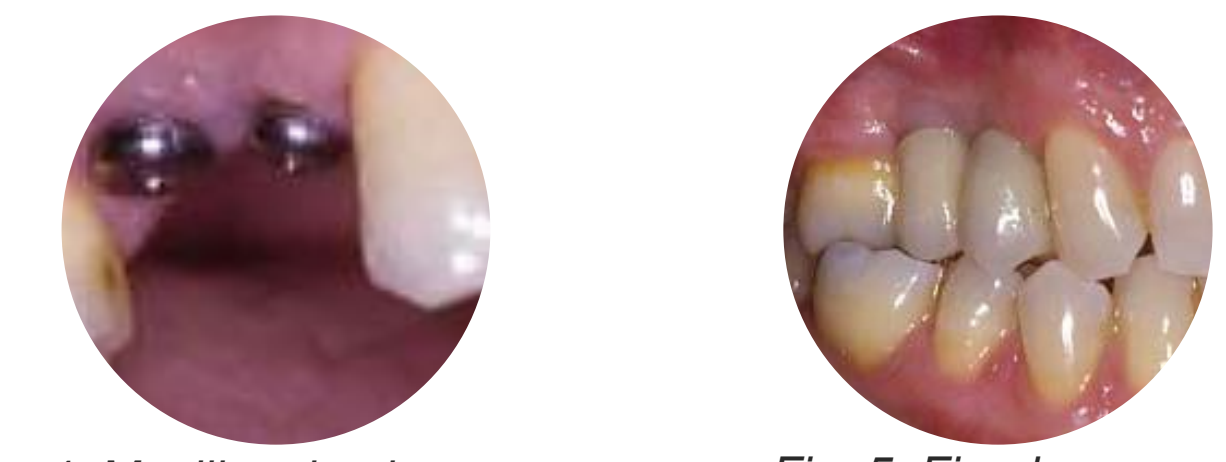

Fig. 4. Maxillary implants
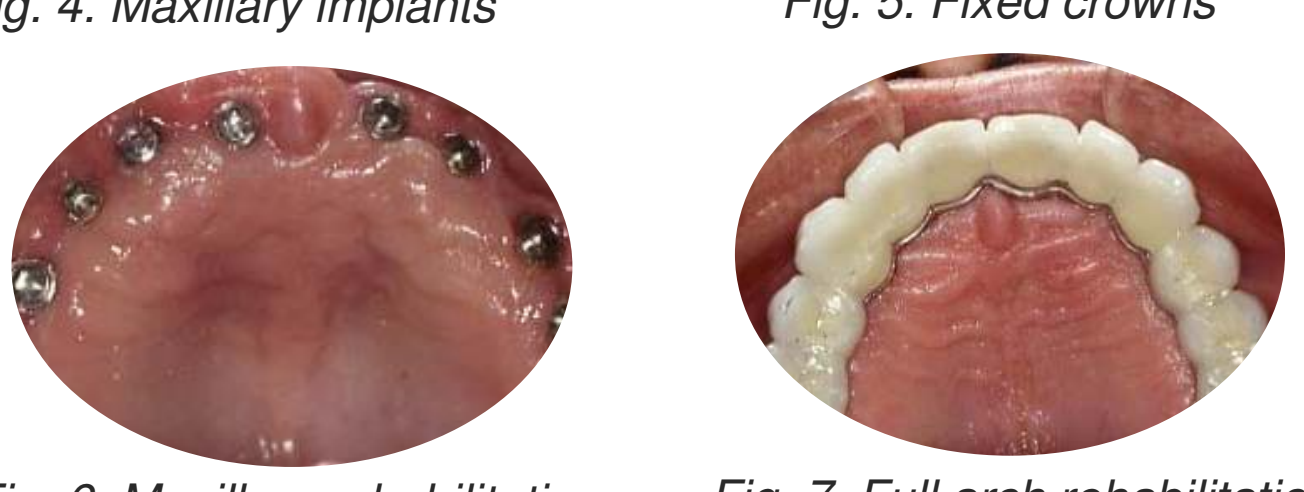

Fig. 7. Full arch rehabilitatio

Fig. 6. Maxillary rehabilitation Fig. 7. Full arch rehabilitation preventive antibiotic regimen (amoxicillin clavulanate) for a week. All patients received loca anesthesia. TSA Defcon ${ }^{\circledR}$ implants (Impladent, Sabadell, Spain) with a acid-etched surface were inserted in a non-submerged surgical protocol, and all were stable after insertion. The basic surgical procedure consisted of a supracrestal incision, with performing full thickness flap with exposure of the underlying alveolar bone. The preparation and insertion of implants was performed according to the conventional standardized protocol with consecutive drills from smallest to largest diameter at a constant speed 800 r.p.m. The soft tissues were sutured around the healing screws (one surgery). A week later, the sutures were removed and patients were
instructed on basic oral hygiene care. Daily instructed on basic oral hygiene care. Daily
chlorhexidine rinse was recommended during the first 30 days.

PROSTHODONTICS. Edentulous patients did not use their full denture for 4 weeks after insertion of the implants. Patients with partial or single edentulism did not use temporary restorations, except in frontal cases. In these cases, the temporary removable restorations were tooth-supported without contact with the implant and occlusion free. After 6 weeks, the implants inserted into the mandible and 8 weeks in the maxilla, prosthetic procedures for the early functional loading of implants was performed. The time from the functional
15 years.

\section{Results}

PATIENTS. 35 patients (23 men and 12 women) participated in the study and were treated with mplants, with a mean age of 46.7 years (range 30-70 ears). 9 total edentulous, 7 partially edentulous and 9 with single loss.

IMPLANTS. A total of 100 implants Defcon TSA were inserted, representing an average of 2.8 implants per patient. About the implants used, 55 were $3.4 \mathrm{~mm}$ in diameter; 45 of $4 \mathrm{~mm}$ diameter. Regarding the length they were inserted 42 implants $10 \mathrm{~mm}, 13 \mathrm{~mm}$ and 58. 47 implants were inserted in the maxilla, while 53 were in the jaw. All implants $(100 \%)$ were inserted in one way surgical phase. In 3 implants $(3 \%)$ had complications. 3 implants showed mobility clinical examination during the functional load-free healing prosthodontic functional loading during the 2 years of clinical follow-up.

Implant supported prosthesis. 97 implants were unctionally loaded with its corresponding prosthetic implant restoration, 51 implants in the jaw (6 weeks) and 46 implants in the maxilla (8 weeks). After the period of osseointegration the following prostheses were made: 37 single crowns, 8 fixed bridges ( 7 with 2 implants and 1 with 3 implants), and 12
overdentures (10 with retention by bars and 2 by overdents).

\begin{tabular}{|cccc|}
\hline Long Term & Patients & $\begin{array}{c}\text { Following } \\
\text { (years) }\end{array}$ & $\begin{array}{c}\text { Success } \\
\text { rate }\end{array}$ \\
\hline $\begin{array}{c}\text { Attard et al } \\
(2003)^{2}\end{array}$ & 130 & 10 & $91,6 \%$ \\
\hline $\begin{array}{c}\text { Ekelund et } \\
\text { al (2003) }\end{array}$ & 47 & 20 & $98,9 \%$ \\
\hline $\begin{array}{c}\text { Branemark } \\
\text { et al } \\
(1995)^{4}\end{array}$ & 156 & 10 & $90,8 \%$ \\
\hline Results & 35 & 15 & $97 \%$ \\
\hline
\end{tabular}

Fig 8. Table comparing long term success rate

\section{Discussion}

Implant treatment has been developed over the last 50 years, but is still limited knowledge of biological and long-term clinical results, because in relation to the numerous publications on oral implantology, there are few studies of 10 or more years.

The pioneering studies of long-term success were made especially in edentulous patients with implants machined surface and functional protocols loaded with a healing period of 3-6 months and hybrid screwed prosthesis, called Branemark protocol or Toronto. ${ }^{5}$

Gothenburg School has offered the first long-term studies that reflected implant survival of $80-98 \%$. A first retrospective study evaluated the clinical outcomes of the complete rehabilitation with 4-6 implants in 156 edentulous patients. They were performed on 4 or 6 implants, 13 and 59 mandibular prosthesis; and jaws 14 and 70 , after a period of 3-8 months conventional healing. After 10 years, the survival rate of implants and prostheses in the mandible was $88.4 \%$ and $93.2 \%$; while the jaw was $80.3 \%$ and $78.3 \%$, respectively. ${ }^{4}$

The timing of the functional load is an important implant treatment of the edentulous maxilla and it will depend on the analysis of a number of factors that have been evaluated in patient preplanning step. In addition, clinical results from surgery can confirm or change the type of functional load as poorer bone quality or use of biological complex techniques (eg GTR ) or surgical (eg . Grafting or sinus elevation) will influence in primary stability of the implant and may recommend delaying the time of its functional load.

Primary stability and absence of micromovement are considered two of the main to obtain predictable results and high success rates in implant factors, since the presence of micro-movements can lead to soft tissue interface that encapsulates the implant. To minimize this risk, a free charging time is recommended during the healing period ( 3 to months in the mandible and 6 to 8 months jaw).

Generally, during that period, patients often use removable dentures that some of them are really uncomfortable. That is why began to look shorten healing of bone anchored implants, being Lederman in 1979 the first author to talk about immediate loading, but not until 1990 when the first longitudinal clinical study on immediate loading was published and early. 6,7

\section{Conclusions}

The clinical findings of this study indicate that prosthodontic rehabilitation with implants with acid etched surface and loaded early, represents successful dental treatment

This success can be maintained over time if proper the follow-up to 15 years.

\section{References}

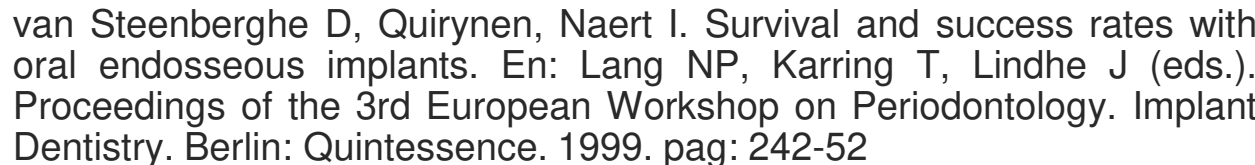
Attard NJ, Zarb GA. Implant prosthodontic management of partially
edentullous patients missing posterior teeth: the Toronto experience. Prosthet Dent 2003; 89:352-359. Ekelund JA, Lindquist LW, Carlsson GE, JJemt T. Implant treatment in the
edentulous mandible: a prospective study on Branemark system implants Branemark PI, Svensson B, van Steenberghe D. Ten-year survival rates
of fixed prostheses on tour or six implants ad modum Branemark in full
edentulism Clin Oral Impl Res 1995; 6-227-231. Attard NJ, Zarb GA. Long-term treatment outcomes in edentulous
patients with implant-fixed prostheses: The Toronto study. In J
Prosthodont 2004: 17:417-424. Testori T, Galli F, Capelli M, Zuffettit F, Esposito M. Immediate non-
occlusal versus early loading of dental implants in partially edentulluous
patients: 1 -year results from a multicenter, randomized controlled clinical . Xu L, Wang X, Zhang Q, Yang W, Zhu W, Zhao K. Immediate versus
early Ioading of flapless placed dental implants: a systematic review.
Prosthet Dent 2014: 112:760-769. 